K. SEKIGAWA AND L. VANHECKE

KODAI MATH. J.

9 (1986), 215-224

\title{
VOLUME-PRESERVING GEODESIC SYMMETRIES ON FOUR-DIMENSIONAL 2-STEIN SPACES
}

\author{
By K. Sekigawa And L. VAnhecke
}

\section{Introduction}

Let $(M, g)$ be an $n$-dimensional Riemannian manifold such that all local geodesic symmetries are volume-preserving (up to sign). Locally symmetric spaces, naturally reductive homogeneous spaces and commutative spaces are examples of such manifolds. (See [14] for more details.) To our knowledge an example which is not locally homogeneous is not known and there is some support for an affirmative answer to the following question:

Are Riemannian manifolds such that all local geodesic symmetries are volumepreserving, locally homogeneous manifolds?

For two- and three-dimensional manifolds this is indeed the case [10], but for $n \geqq 4$, it is still an open problem.

In [12], we started our research about this question for four-dimensional manifolds and proved that four-dimensional Kähler manifolds with volumepreserving geodesic symmetries are locally symmetric. (See also [11].) On the other hand, a well-known theorem of G.R. Jensen [8] states that any fourdimensional locally homogeneous Einstein space is locally symmetric. In view of this result, it is worthwhile to consider the open problem for the class of Einstein spaces.

In this paper we study this problem for a particular subclass of Einstein spaces, namely the so-called 2-stein spaces [2]. In this way we answer the problem stated at the end of [12]. More precisely, we prove:

MAIN THEOREM. Let $(M, g)$ be a connected four-dimensional 2-stein space with volume-preserving local geodesic symmetries. Then $(M, g)$ is locally flat or locally isometric to a rank one symmetric space.

This theorem is a generalization of the theorem of Lichnerowicz and Walker about four-dimensional harmonic spaces (see for example [1], p. 166).

The paper is organized as follows. In section 2 we first consider general Riemannian manifolds with volume-preserving local geodesic symmetries. Then, in section 3, we write down some useful facts about the special geometry of four-dimensional Einstein spaces. Finally, the proof of the Main Theorem is

Received October 19, 1985 
given in section 4 .

\section{Volume-Preserving Geodesic Symmetries}

Let $(M, g)$ be an $n$-dimensional Riemannian manifold of class $C^{\infty}, m$ a point of $M$ and $T_{m} M$ the tangent space of $M$ at $m$. For a unit vector $\xi \in T_{m} M$ we denote by $\gamma: r \mapsto \exp _{m}(r \xi)$ the geodesic of $M$ with arc length $r$. We always suppose $r<i(m)$, the injectivity radius at $m$.

Next, let $\left\{e_{\imath}, i=1, \cdots, n\right\}$ be an orthonormal frame at $m$ and let $\left(x_{1}, \cdots, x_{n}\right)$ be a system of normal coordinates centered at $m$ and such that $\frac{\partial}{\partial x_{\imath}}(m)=e_{\imath}$ for $i=1, \cdots, n$. Then the volume density function $\theta_{m}$ of $\exp _{m}$ is given by

$$
\theta_{m}=\left(\operatorname{det}\left(g_{\imath j}\right)\right)^{1 / 2}
$$

where $g_{\imath \jmath}=g\left(\frac{\partial}{\partial x_{\imath}}, \frac{\partial}{\partial x_{\jmath}}\right)$.

Let $p=\exp _{m}(r \xi)$. Then we have the following Taylor expansion [3], [6], [7]:

$$
\theta_{m}(p)=1+\sum_{k=2}^{7} \alpha_{k}(m, \xi) r^{k}+0\left(r^{8}\right),
$$

where the $\alpha_{k}$ are completely determined by the Riemann curvature tensor and its covariant derivatives. In particular we have

$$
\left\{\begin{aligned}
\alpha_{2}(m, \xi)= & -\frac{1}{6} \rho_{\xi \xi}(m), \\
\alpha_{3}(m, \xi)= & -\frac{1}{12}\left(\nabla_{\xi} \rho_{\xi \xi}\right)(m), \\
\alpha_{4}(m, \xi)= & \frac{1}{24}\left(-\frac{3}{5} \nabla_{\xi \xi}^{2} \rho_{\xi \xi}+\frac{1}{3} \rho_{\xi \xi}^{2}-\frac{2}{15} \sum_{a, b=1}^{n} R_{\xi a \xi b}^{2}\right)(m), \\
\alpha_{5}(m, \xi)= & \frac{1}{120}\left(-\frac{2}{3} \nabla_{\xi \xi \xi}^{3} \rho_{\xi \xi}+\frac{5}{3} \rho_{\xi \xi} \nabla_{\xi} \rho_{\xi \xi}-\frac{2}{3} \sum_{a, b=1}^{n} R_{\xi a \xi b} \nabla_{\xi} R_{\xi a \xi b}\right)(m), \\
\alpha_{6}(m, \xi)= & \frac{1}{720}\left(-\frac{5}{7} \nabla_{\xi \xi \xi \xi}^{4} \rho_{\xi \xi}+3 \rho_{\xi \xi} \nabla_{\xi \xi}^{2} \rho_{\xi \xi}+\frac{5}{2}\left(\nabla_{\xi} \rho_{\xi \xi}\right)^{2}\right. \\
& -\frac{5}{9} \rho_{\xi \xi}^{3}-\frac{8}{7} \sum_{a, b=1}^{n} R_{\xi a \xi b} \nabla_{\xi \xi}^{2} R_{\xi a \xi b}-\frac{15}{14} \sum_{a, b=1}^{n}\left(\nabla_{\xi} R_{\xi a \xi b}\right)^{2} \\
& \left.-\frac{16}{63} \sum_{a, b, c=1}^{n} R_{\xi a \xi b} R_{\xi b \xi c} R_{\xi c \xi a}+\frac{2}{3} \rho_{\xi \xi} \sum_{a, b=1}^{n} R_{\xi a \xi b}^{2}\right)(m) .
\end{aligned}\right.
$$

Here $\nabla$ denotes the Levi Civita connection, $R$ is the Riemann curvature tensor and $\rho$ the corresponding Ricci tensor. $\alpha_{7}$ is still more complicated but it can be given by the following formula (see [14]): 


$$
2 \alpha_{7}(m, \xi)=\sum_{\imath=2}^{6} \frac{(-1)^{\imath}}{(7-i) !}\left(\nabla_{\xi \cdots \xi}^{7-\imath} \alpha_{\imath}\right)(m, \xi)
$$

The local geodesic symmetry $\varphi_{m}$ at $m$ is defined by $\varphi_{m}: \exp _{m}(r \xi) \mapsto \exp _{m}(-r \xi)$ and it is a local diffeomorphism. It preserves the volume (up to sign) if and only if

$$
\theta_{m}\left(\exp _{m}(r \xi)\right)=\theta_{m}\left(\exp _{m}(-r \xi)\right)
$$

for allunit vectors $\xi \in T_{m} M$. (See [4].) Hence, we have

LEMMA 1. Let $(M, g)$ be a Riemannian manifold of class $C^{\infty}$ such that all local geodesic symmetries are volume-preserving (up to sign). Then we have at each $m \in M$ and for any $\xi \in T_{m} M$ :

$$
\alpha_{2 k+1}(m, \xi)=0, \quad k \in \boldsymbol{N}_{0} .
$$

Note that the conditions (3) are also sufficient when $(M, g)$ is analytic. The first condition is equivalent to

$$
\nabla_{\xi} \rho_{\xi \xi}=0 .
$$

J. L. Kazdan has communicated to the second author that a Riemannian manifold of class $C^{\infty}$, satisfying (4), is always analytic in geodesic normal coordinates. (See [5] for the Einstein case.) So, there is no restriction when we suppose the manifold to be analytic.

(4) is always satisfied for Einstein spaces. Next, we consider the second condition, taking into account the condition (4). Then, $\alpha_{5}(m, \xi)=0$ is equivalent to

$$
\sum_{a, b=1}^{n} R_{\xi a \xi b} \nabla_{\xi} R_{\xi a \xi b}=0 .
$$

To derive a condition from this, and in the rest of this paper, we shall adapt the following notation:

$$
F(\xi)=\sum_{a, b=1}^{n} R_{\xi a \xi b}^{2}, \quad G(\xi)=\sum_{a, b=1}^{n} R_{\xi a \xi b} \nabla_{\xi} R_{\xi a \xi b},
$$

for all $\xi \in T_{m} M$ and all $m \in M$. Then we may regard $F$ and $G$ as differentiable functions on the tangent bundle. Next, we identify, for each $m \in M$, the tangent space $T_{m} M$ with an $n$-dimensional Euclidean space $\boldsymbol{R}^{n}$ via an orthonormal basis of $T_{m} M$. Then we may regard the restrictions of $F$ and $G$ to $m$ as homogeneous polynomials of degree 4 and 5 respectively. Finally, we denote by $D$ the Laplacian of $\boldsymbol{R}^{n}$. Then we have [12]

LEMMA 2. Let $(M, g)$ be a Ricci-parallel manifold (i.e. $\nabla \rho=0)$. Then we have at each point $m \in M$

$$
\left(D^{2} G\right)(\xi)=12 \xi\|R\|^{2}
$$


Hence we obtain

COROLlary 3. Let $(M, g)$ be a connected Einstern space such that all local geodesic symmetries are volume-preserving. Then $\|R\|^{2}$ is constant on $(M, g)$.

Finally, it is worthwhile to note the following

PROPOSITION 4 [3]. Let $(M, g)$ be an $n$-dimensional $(n>2)$ reducible manifold such that for each $m \in M, F(\xi)$ is independent of $\xi$, for all unit vectors $\xi \in T_{m} M$. Then $(M, g)$ is locally flat.

\section{Einstein Manifolds of Dimension Four}

Before we consider the third condition $\left(\alpha_{7}=0\right)$, we suppose that $(M, g)$ is a four-dimensional Einstein space, and we write down some well-known facts about the special features of the geometry on such manifolds.

Following [1], [13] we can connect with any fixed point $m \in M$ a so-called Singer-Thorpe basis $\left\{e_{1}, e_{2}, e_{3}, e_{4}\right\}$ of $T_{m} M$. This is an orthonormal basis such that all the components of the curvature tensor $R$ are given by the following formulas :

$$
\left\{\begin{array}{l}
R_{1212}=R_{3434}=a, \quad R_{1313}=R_{2424}=b, \quad R_{1414}=R_{2323}=c, \\
R_{1234}=\alpha, \quad R_{1342}=\beta, \quad R_{1423}=\gamma, \\
R_{\imath j k l}=0 \text { whenever just three of the indices } i, j, k, l \text { are distinct. }
\end{array}\right.
$$

Note that $\alpha+\beta+\gamma=0$ (the first Bianchi identity) and

$$
a+b+c=\frac{\tau}{4}
$$

where $\tau$ denotes the scalar curvature. Moreover it is always possible to choose a Singer-Thorpe basis such that $a=\max \sigma$ and $c=\min \sigma$, where $\sigma$ denotes the sectional curvature function at $m$.

Further we write down some useful expressions for some curvature invariants of order 2 and 3 with respect to a Singer-Thorpe basis. We have (see [9])

$$
\left\{\begin{array}{l}
\|R\|^{2}=8\left(a^{2}+b^{2}+c^{2}+\alpha^{2}+\beta^{2}+\gamma^{2}\right), \\
\check{R}=\sum R_{\imath j k l} R_{k l p q} R_{p q \imath \jmath}=16\left(a^{3}+b^{3}+c^{3}+3 a \alpha^{2}+3 b \beta^{2}+3 c \gamma^{2}\right), \\
\check{R}=\sum R_{i k j l} R_{k p l q} R_{p q q \jmath}=24(a b c+a \beta \gamma+b \alpha \gamma+c \alpha \beta) .
\end{array}\right.
$$

Further, we have

$$
\langle\Delta R, R\rangle=\frac{1}{2} \Delta\|R\|^{2}-\|\nabla R\|^{2}
$$

and (see [7]) 


$$
\left\{\begin{array}{l}
\check{R}=-\frac{\tau^{3}}{12}+\frac{2}{3} \tau\|R\|^{2}-\frac{1}{3}\langle\Delta R, R\rangle, \\
\check{\bar{R}}=\frac{\tau^{3}}{48}-\frac{1}{24} \tau\|R\|^{2}-\frac{1}{6}\langle\Delta R, R\rangle .
\end{array}\right.
$$

Next, let $W \in C^{\infty}\left(\right.$ End $\left.\Lambda^{2} M\right)$ be the Weyl conformal tensor. Then we have the following unpublished result of A. Derdziński :

Proposition 5. Let $(M, g)$ be a four-dimensional Einstein manfold such that $W \in C^{\infty}\left(\right.$ End $\left.\Lambda^{2} M\right)$ has constant eigenvalues. Then $(M, g)$ is locally symmetric.

It is clear :that $W$ has constant eigenvalues if and only if $a, b, c, \alpha, \beta, \gamma$ are constant. As we noted in [12] this is equivalent to the curvature homogeneity of $(M, g)$. So, Proposition 5 may be formulated as

Proposition 6. Let $(M, g)$ be a four-dimensional curvature homogeneous Einstein manifold. Then $(M, g)$ is locally symmetric.

In what follows we shall only consider the subclass of 2-stein spaces. Such manifolds are defined in [2] as Einstein spaces satisfying the additional condition

$$
\text { " } F(x)=\sum_{a, b=1}^{n} R_{x a x b}^{2} \text { is independent of the unit vector } x \in T_{m} M \text { for all } m \in M . "
$$

For 4-dimensional 2-stein spaces we have [12]

LEMmA 7. Let $(M, g)$ be a four-dimensional Einstein space. Then $(M, g)$ is a 2-stein space if and only if

$$
\pm \alpha=a-\frac{\tau}{12}, \quad \pm \beta=b-\frac{\tau}{12}, \quad \pm \gamma=c-\frac{\tau}{12},
$$

for each $m \in M$.

For this class of manifolds we also have some important and useful freedom in the choice of a Singer-Thorpe basis. Indeed, let $I, J, K$ be a quaternionic structure on $T_{m} M$ adapted to the given Singer-Thorpe basis. This means, for

we put

$$
x=a^{1} e_{1}+a^{2} e_{2}+a^{3} e_{3}+a^{4} e_{4},
$$

$$
\begin{aligned}
& I x=-a^{2} e_{1}+a^{1} e_{2}-a^{4} e_{3}+a^{3} e_{4}, \\
& J x=-a^{3} e_{1}+a^{4} e_{2}+a^{1} e_{3}-a^{2} e_{4}, \\
& K x=-a^{4} e_{1}-a^{3} e_{2}+a^{2} e_{3}+a^{1} e_{4} .
\end{aligned}
$$

Then we have [9], [12]

LEMMA 8. For any unit vector $x \in T_{m} M,\{x, I x, J x, K x\}$ is a Singer-Thorpe 
basis if and only if $(M, g)$ is a 2-stein space.

Finally we note that on any 4-dimensional Einstein space we have

$$
\sum_{a, b, c=1}^{4} R_{\xi a b c}^{2}=\frac{1}{4}\|R\|^{2}
$$

for any unit vector $\xi \in T_{m} M$. (See [1] or use a Singer-Thorpe basis to check (13).) For 2-stein spaces we have more. Indeed, using (12) and Lemma 8, we obtain

$$
\left\{\begin{array}{c}
\sum_{\imath, \jmath, k, l, p} R_{\imath j k l} R_{\imath \jmath p \xi} R_{k l p \xi}=\frac{1}{4} \check{R}, \\
\sum_{\imath, \jmath, k, l, p} R_{i k j l} R_{k p l \xi} R_{p i \xi \jmath}=\frac{1}{4} \check{\bar{R}}
\end{array}\right.
$$

for all unit vectors $\xi \in T_{m} M$.

\section{Proof of the Main Theorem}

Now we suppose that $(M, g)$ is a four-dimensional connected 2-stein space with volume-preserving geodesic symmetries. Using the fact that $\|R\|^{2}$ is constant (Corollary 3) and (8), (9), (10), (11), (12), we obtain

$$
\left\{\begin{array}{l}
a+b+c=\frac{\tau}{4}, \\
a b+b c+c a=-\frac{1}{32}\|R\|^{2}+\frac{5}{192} \tau^{2}, \\
96 a b c=\frac{\tau^{3}}{12}+\frac{1}{6}\|\nabla R\|^{2}-\frac{1}{6} \tau\|R\|^{2} .
\end{array}\right.
$$

In what follows we will prove that $\|\nabla R\|^{2}$ is constant. Then, (15) implies that $a, b, c$ are constant, and hence also $\alpha, \beta, \gamma$. So, $(M, g)$ is curvature homogeneous. Proposition 6 then implies that $(M, g)$ is locally symmetric. The required result finally follows as in [1], [9].

To prove that $\|\nabla R\|^{2}$ is constant, we consider the condition

$$
\alpha_{7}(m, \xi)=0 .
$$

We get from (2) and (5) that this is equivalent to

or

$$
\nabla_{\xi} \alpha_{6}=0
$$

$$
\begin{aligned}
8 \sum_{a, b} \nabla_{\xi} R_{\xi a \xi b} \nabla_{\xi \xi}^{2} R_{\xi a \xi b} & +8 \sum_{a, b} R_{\xi a \xi b} \nabla_{\xi \xi \xi}^{3} R_{\xi a \xi b}+15 \sum_{a, b} \nabla_{\xi} R_{\xi a \xi b} \nabla_{\xi \xi}^{2} R_{\xi a \xi b} \\
& +\frac{16}{3} \sum_{a, b, c} R_{\xi b \xi c} R_{\xi c \xi a} \nabla_{\xi} R_{\xi a \xi b}=0
\end{aligned}
$$


Using again (5), (17) becomes

$$
3 \sum_{a, b} \nabla_{\xi} R_{\xi a \xi b} \nabla_{\xi \xi}^{2} R_{\xi a \xi b}=16 \sum_{a, b, c} R_{\xi b \xi c} R_{\xi c \xi a} \nabla_{\xi} R_{\xi a \xi b}
$$

or

$$
3 K(\xi)=16 L(\xi)
$$

where

$$
\begin{aligned}
& K(\xi)=\sum_{a, b} \nabla_{\xi} R_{\xi a \xi b} \nabla_{\xi \xi}^{2} R_{\xi a \xi b}, \\
& L(\xi)=\sum_{a, b, c} R_{\xi b \xi c} R_{\xi c \xi a} \nabla_{\xi} R_{\xi a \xi b},
\end{aligned}
$$

for all $\xi \in T_{m} M$ and all $m \in M$.

Using again the Laplacian on $T_{m} M$, we get from (19)

$$
3\left(D^{3} K\right)(\xi)=16\left(D^{3} L\right)(\xi) \text {. }
$$

First we compute $\left(D^{3} L\right)(\xi)$. Using a Singer-Thorpe basis and Lemma 8, we get

$$
\sum_{a, b, c} R_{\xi a \xi b} R_{\xi b \xi c} R_{\xi c \xi a}=\left(a^{3}+b^{3}+c^{3}\right)\|\xi\|^{6}
$$

for all $\xi \in T_{m} M$ and with (15) this becomes

$$
\sum R_{\xi a \xi b} R_{\xi b \xi c} R_{\xi c \xi a}=\frac{1}{192}\left(\|\nabla R\|^{2}+\frac{7}{2} \tau\|R\|^{2}-\frac{1}{4} \tau^{3}\right)\|\xi\|^{6} .
$$

Hence, we have from (21)

$$
L(\xi)=\frac{1}{576}\|\xi\|^{6} \xi\|\nabla R\|^{2}
$$

for all $\xi \in T_{m} M$ and all $m \in M$. By direct calculation we obtain from (22)

$$
\left(D^{3} L\right)(\xi)=40 \xi\|\nabla R\|^{2} .
$$

The computation of $D^{3} K$ is much more complicated. We shall need several formulas which we write down in the following lemma. They may be obtained by using the Bianchi identities, (10), (11), (13), (14). We omit the long and tedious calculations.

LEMma 9. Under the hypotheses of the Man Theorem, we have the following identıties:

$$
\begin{aligned}
& \Sigma \nabla_{\jmath} R_{\imath a k b} \nabla_{j k}^{2} R_{\imath a \xi b}=\frac{1}{4} \xi\|\nabla R\|^{2}+\frac{1}{6} \xi \check{R}=\frac{11}{36} \xi\|\nabla R\|^{2}, \\
& \Sigma \nabla_{j} R_{\imath a k b} \nabla_{j \xi}^{2} R_{\imath a k b}=\frac{1}{2} \xi\|\nabla R\|^{2}+\frac{1}{3} \xi \check{R}=\frac{11}{18} \xi\|\nabla R\|^{2}, \\
& \Sigma \nabla_{j} R_{\imath a k b} \nabla_{k j}^{2} R_{\imath a \xi b}=\frac{1}{4} \xi\|\nabla R\|^{2}+\frac{1}{4} \xi \check{R}=\frac{1}{3} \xi\|\nabla R\|^{2},
\end{aligned}
$$




$$
\sum \nabla_{j} R_{\imath a \xi b} \nabla_{k j}^{2} R_{\imath a k b}=-\frac{1}{12} \xi \check{R}-\frac{1}{3} \xi \check{\bar{R}}=-\frac{1}{12} \xi\|\nabla R\|^{2},
$$$$
\sum \nabla_{\xi} R_{k b i a} \nabla_{j j}^{2} R_{k b i a}=-\frac{1}{3} \xi \check{R}-\frac{4}{3} \xi \check{\bar{R}}=-\frac{1}{3} \xi\|\nabla R\|^{2},
$$$$
\sum \nabla_{j} R_{k a \xi b} \nabla_{\imath j}^{2} R_{\imath a k b}=-\frac{1}{24} \xi\|\nabla R\|^{2},
$$

for all $\xi \in T_{m} M$ and all $m \in M$.

Using Lemma 9 we then obtain

LEMMA 10. Under the hypotheses of the Main Theorem, we have

$$
(D K)(\xi)=\left\{2 f_{1}+2 f_{2}+10 f_{3}+4 f_{4}+4 f_{5}+2 f_{6}\right\}(\xi)
$$

where

$$
\begin{aligned}
& f_{1}(\xi)=\Sigma \nabla_{\imath} R_{\xi a \xi b} \nabla_{i \xi}^{2} R_{\xi a \xi b}, \\
& f_{2}(\xi)=\Sigma \nabla_{\imath} R_{\xi a \xi b} \nabla_{\xi i}^{2} R_{\xi a \xi b}, \\
& f_{3}(\xi)=\Sigma \nabla_{\xi} R_{\imath a \xi b} \nabla_{\xi \xi}^{2} R_{\imath a \xi b}, \\
& f_{4}(\xi)=\Sigma \nabla_{\xi} R_{\imath a \xi b} \nabla_{i \xi}^{2} R_{\xi a \xi b}, \\
& f_{5}(\xi)=\Sigma \nabla_{\xi} R_{\xi a \xi b} \nabla_{i \xi}^{2} R_{\imath a \xi b}, \\
& f_{6}(\xi)=\Sigma \nabla_{\xi} R_{\xi a \xi b} \nabla_{\imath \imath}^{2} R_{\xi a \xi b}
\end{aligned}
$$

and

$$
\begin{cases}\left(D^{2} f_{1}\right)(\xi)=\frac{44}{3} \xi\|\nabla R\|^{2}, & \left(D^{2} f_{4}\right)(\xi)=\frac{92}{9} \xi\|\nabla R\|^{2}, \\ \left(D^{2} f_{2}\right)(\xi)=12 \xi\|\nabla R\|^{2}, & \left(D^{2} f_{5}\right)(\xi)=-4 \xi\|\nabla R\|^{2}, \\ \left(D^{2} f_{3}\right)(\xi)=\frac{112}{9} \xi\|\nabla R\|^{2}, & \left(D^{2} f_{6}\right)(\xi)=-8 \xi\|\nabla R\|^{2}\end{cases}
$$

for all $\xi \in T_{m} M$ and all $m \in M$. 
Now we can finish the proof. Indeed, from Lemma 10, we obtain

$$
\left(D^{3} K\right)(\xi)=\frac{560}{3} \xi\|\nabla R\|^{2} .
$$

Hence, (19), (23) and (35) give

$$
7 \xi\|\nabla R\|^{2}=8 \xi\|\nabla R\|^{2}
$$

or

$$
\xi\|\nabla R\|^{2}=0 .
$$

Since $(M, g)$ is connected, $\|\nabla R\|^{2}$ is constant. So, we obtain the required result.

\section{REFERENCES}

[1] A.L. BEsse, Manifolds all of whose geodesics are closed, Ergebnisse der Mathematik, 93, Springer-Verlag, Berlin, 1978.

[2] P. Carpenter, A. Gray and T.J. Willmore, The curvature of Einstein symmetric spaces, Quart. J. Math. Oxford 33 (1982), 45-64.

[3] B.Y. Chen and L. Vanhecke, Differential geometry of geodesic spheres, $J$. Reine Angew. Math. 325 (1981), 28-67.

[4] J.E. D'Atri AND H.K. Nickerson, Divergence-preserving geodesic symmetries, J. Differential Geometry 3 (1969), 467-476.

[5] D.M. Deturck And J.L. Kazdan, Some regularity theorems in Riemannian geometry, Ann. Scient. Éc. Norm. Sup. 14 (1981), 249-260.

[6] A. Gray, The volume of a small geodesic ball in a Riemannian manifold, Michigan Math. J. 20 (1973), 329-344.

[7] A. Gray And L. Vanhecke, Riemannian geometry as determined by the volumes of small geodesic balls, Acta Math. 142 (1979), 157-198.

[8] G. R. JENSEN, Homogeneous Einstein spaces of dimension four, J. Differential Geometry 3 (1969), 309-349.

[9] O. Kow ALSKI AND L. VANHECKE, Ball-homogeneous and disk-homogeneous Riemannian manifolds, Math. Z. 180 (1982), 429-444.

[10] O. KowALsKI, Spaces with volume-preserving symmetries and related classes of Riemannian manifolds, Rend. Sem. Mat. Univ. e Politec. Torino, to appear.

[11] K. Sekigawa and L. VANHEcke, Symplectic geodesic symmetries on Kähler manifolds, Quart. J. Math. Oxford, to appear.

[12] K. SEKIGAWA AND L. VANHECKE, Volume-preserving geodesic symmetries on four-dimensional Kähler manifolds, to appear.

[13] I. M. Singer AND J. A. Thorpe, The curvature of 4-dimensional Einstein spaces, in Global Analysis (Papers in honor of K. Kodaira), Princeton University Press, Princeton 1969, 355-366.

[14] L. VAnHecke, A note on harmonic spaces, Bull. London Math. Soc. 13 (1981), $545-546$.

[15] L. VANHECKE, Some results about homogeneous structures on Riemannian manifolds, Differential Geometry, Proc. Conf. Differential Geometry and its Applications, Nové Město 1983, Univerzita Karlova, Praha, 1984, 147-164. 
Department of Mathematics Department of Mathematics Nilgata University, Katholieke Universiteit Leuven NIIGATA, 950-21, JAPAN CelestijnenlaAn 200B B-3030 Leuven, Belgium. 